\title{
Clouds at CTIO and the Dark Energy Survey
}

\author{
Eric H. Neilsen, Jr.*
}

\section{Abstract}

An understanding of the weather patters at Cerro-Tololo Inter-American (CTIO) Observatory, the observing site for the Dark Energy Survey (DES), is important for assessing the efficiency of DES operations in using observing time and for planning future operations. CTIO has maintained records of cloud-cover by quarters of nights since 1975 . A comparison between these cloud records in the 2013-2014 DES observing season (DES year 1) and achieved observing efficiency and exposure quality allows the DES collaboration to make better use of the historical records in survey planning. Plots and tables here relate human recorded cloud-cover to collection of good DES data, show the variation of typical cloud-cover by month, and evaluate the relationship between the El Niño weather pattern and cloud-cover at CTIO.

\section{Introduction}

The Dark Energy Survey (DES)[Flaugher (2005)] is an astronomical survey being conducted using the DECam imaging camera[[Flaugher et al. (2015)] mounted on the Blanco telescope at Cerro Tololo Inter-American Observatory (CTIO). The survey began in August of 2013, and will use 105 nights between August and February each year for five years, for a total of 525 nights [Diehl et al. (2014)].

To understand what time is required to collect the data needed to reach our science goals (or, conversely, what data can be collected given a specific observing schedule), an estimate of how much time will be lost to cloudy weather is essential.

CTIO has a $\sim 50$ year history of use as an astronomical observatory [KPNO-CTIO (1966)], and historical records taken by the observatory provide a basis for estimating the time useful for astronomical observing. The correspondence between time useful for DES and the human-estimated cloud cover values recorded at CTIO was not, however, obvious, and represents a source of uncertainty in survey design and planning.

${ }^{*}$ Fermi National Accelerator Laboratory, P. O. Box 500, Batavia, IL 60510 , USA
This correspondence can be measured using data from the first year of DES observing, during which human observations continued to be recorded, and the effect on DES observing can be measured directly.

\section{Sources of information}

\subsection{Historical records from CTIO}

Observers at each of the telescopes at CTIO record the conditions in each quarter of each night in eighths cloud-cover, where 0 represents photometric (completely clear) and 8 represents completely overcast ${ }^{1}$ The observatory staff then averages the values recorded by the observers at the different telescopes and produces a record of the cloud cover for each quarter of each night[CTIO (2015)]. Such records are currently available for dates between January 1, 1975 and March 31,2014 , a range which includes the first but not second year of DES observing. Of the 57344 quarter-nights in this range, $56003(98 \%)$ have cloud-cover data.

\subsection{Completed DES exposures}

During each night of scheduled DES observing, DES observers used DECam to collect science data, weather permitting. DES data management processes this data and generates metrics used to evaluate the quality of exposures in order to determine which observations need to be repeated on a future night. Among these metrics is $t_{\text {eff }}$, a factor which scales the exposure time of the collected image to the exposure time needed to achieve the same signal to noise under "good" observing conditions, defined to be a dark (moonfree, cloud-free) skies, at zenith, with a seeing of $0.9^{\prime \prime}$ in i band[Neilsen et al. (2015)]. For example, an exposure with a measured $t_{\text {eff }}=0.5$ and an exposure time of 90 seconds has the same $\mathrm{S} / \mathrm{N}$ of a point source as a hypothetical exposure of 45 seconds taken under these nominal "good" conditions.

The DES-DM database provides these $t_{\text {eff }}$ values for each DES science exposure to the DES collaboration.

\footnotetext{
${ }^{1} \mathrm{~A} 9$ represents missing data.
} 


\section{Recorded clouds and useful exposure time}

Figures 1 shows the distribution of observing efficiency, $\left(\sum t_{\text {eff }} \times\right.$ EXPTIME $)$ in seconds of normalized exposure time per hour of observing, which corresponds to the $\mathrm{S} / \mathrm{N}$ that could be gathered in an an hour of typical DES exposures. Figure 2 shows fraction of time spent with the shutter open, taking exposures with data quality usable for DES, as a function of human-recorded cloud levels. The values plotted appear in tables 1 and 2 normalized to clear conditions. The distributions for 1 through 4 appear nearly indistinguishable; these values are designated "partly cloudy" in figures 3. 4,5 and 6 . The distributions for 5 and 6 eighths appear similar to each other, and are designated "mostly cloudy" in these plots. Exposures taken in quarters rated 7 or 8 were rarely useful.

Table 1: The fraction of time spent with "shutter open" on good science exposures relative to clear conditions.

\begin{tabular}{rr} 
clouds & relative fraction open shutter time \\
\hline 0 & 1.00 \\
1 & 0.77 \\
2 & 0.75 \\
3 & 0.69 \\
4 & 0.67 \\
5 & 0.36 \\
6 & 0.25
\end{tabular}

Table 2: The total exptime $\times t_{\text {eff }}$ relative to clear conditions.

\begin{tabular}{rr} 
clouds & relative total exptime $\times t_{\text {eff }}$ \\
\hline 0 & 1.00 \\
1 & 0.68 \\
2 & 0.66 \\
3 & 0.52 \\
4 & 0.62 \\
5 & 0.29 \\
6 & 0.20
\end{tabular}

Figure 3 shows the history of each quality of time for recorded half nights in the CTIO records. Not all years have the same total "height" because different years have different numbers of quarters with no data. ${ }^{2}$

\footnotetext{
${ }^{2}$ No attempt has been made to normalize these bars to a common height, because such a scaling is not straightforward: periods for which there is no data sometimes cover a month or more (see the 1989-1990, 1992-1993, 19961997, and 2005-2006 seasons in figure 5. The cloud distribution these might have been depends on which time of year (see figure 4 these periods fall in:
}

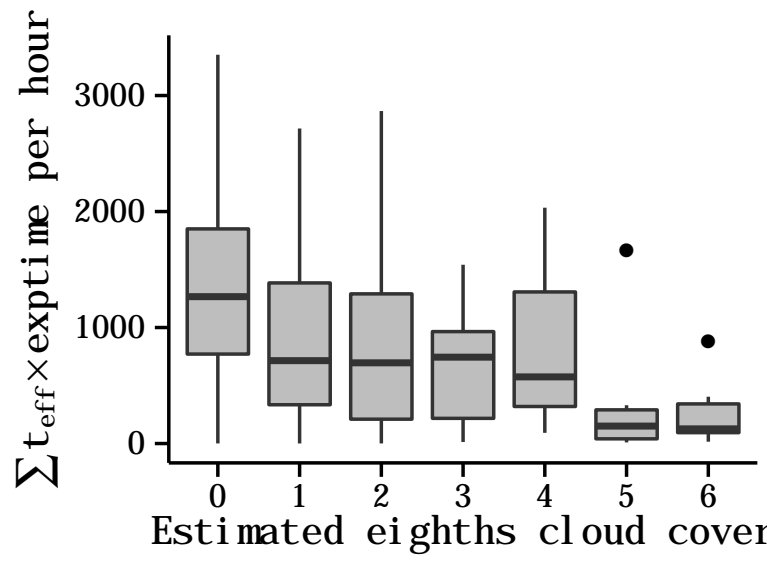

Figure 1: The delivered distribution of observing efficiency $\left(\sum t_{\text {eff }} \times\right.$ EXPTIME $)$ in seconds of exposure time per hour of observing as a function of human recorded cloud levels. The gray box marks the second and third quartiles, and the central horizontal bar marks the median. The whiskers extend from the lowest to highest data points within 1.5 times the inter-quartile range (distance between first and third quartile), and points representing individual measurements falling outside this range [McGill, Tukey, \& Larsen (1978)].

Figure 4 splits each year up by month, and figure 5 shows the distribution in each month, combining all data. These data show trend in which months later in the DES observing season generally have a higher fraction of useful time, with a transition from worse to better months occurring in October or November.

\section{El Niño and clouds at CTIO}

Cloud cover at CTIO is reputed to be correlated with the El Niño weather pattern. Figure 6 plots the distributions of weather conditions in different ranges of Oceanic Niño Index (ONI), the metric used by the National Oceanic and Atmospheric Administration (NOAA) to define La Niña and El Niño events ${ }^{3}$ obtained from the NOAA website.[NOAA CPC (2014)] Figure 7 plots numbers of photometric quarter-nights (human reported eighths cloud coven of 0 ) agains the mean ONI for the August-February

\footnotetext{
all simple options for re-scaling (assuming a constant value or renomalizing to a common height) are obviously inappropriate.

${ }^{3}$ The ONI is the difference between the mean surface temperature (averaged over three months and a set of locations) of the Pacific ocean and the thirty-year average. The NOAA defines El Niño events to be periods where the ONI exceeds 0.5 degrees for " 5 consecutive overlapping seasons"[NOAA CPC (2014)].
} 


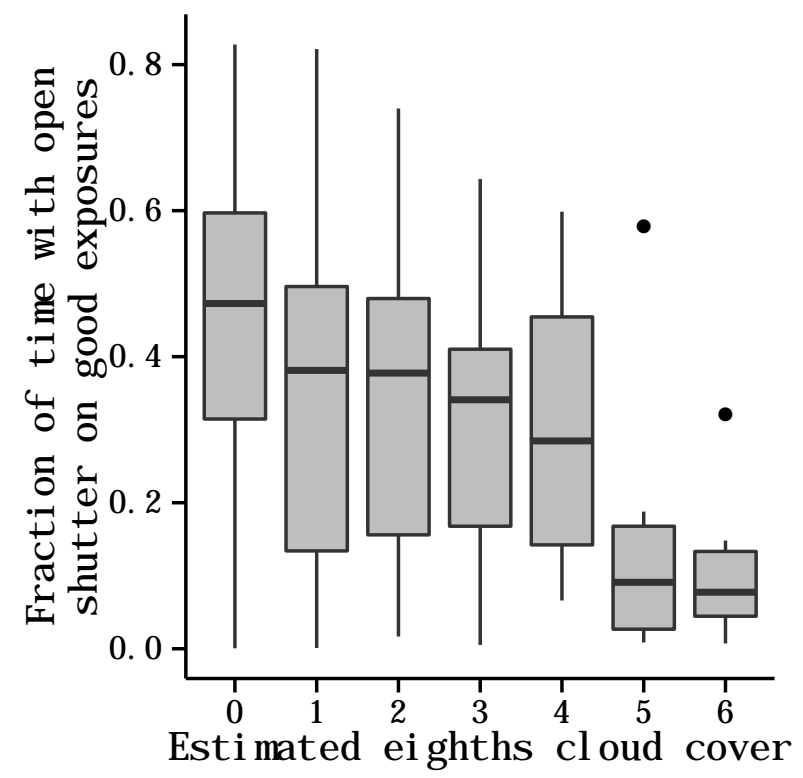

Figure 2: The fraction of time spent with the shutter open on exposures eventually found to be useful for DES, as a function of human recorded cloud levels. The gray box marks the second and third quartiles, and the central horizontal bar marks the median. The whiskers extend from the lowest to highest data points within 1.5 times the inter-quartile range (distance between first and third quartile), and points representing individual measurements falling outside this range.

periods of the available data, excluding years where there large numbers of quarter-nights without data. The blue line shows a least squares fit. The slope of this fit is $-5.5 \% \pm 0.1 \%$ per degree, a $5.5 \sigma$ deviation from 0 , suggesting that the ONI has a significant effect on observing conditions.

\section{Conclusion}

Examination of DES exposure data quality and historical records of cloud-cover at CTIO reveals several factors important for estimating future DES data colletion rates:

- Human estimates of eighths cloud-cover can merged into four effective categories corresponding to clear, partly cloudy, mostly cloudy, and overcast. Different recorded valuse of eighths result in similar observing efficiency for all members of the same class.

- On a typical year, a higher fraction of observing time will be lost to clouds early in the DES observing season

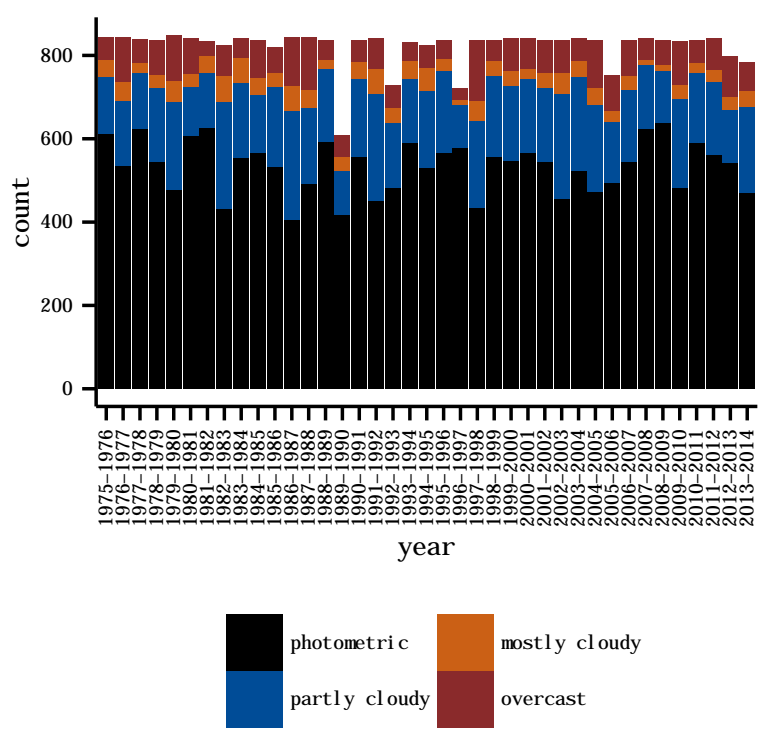

Figure 3: The distribution of cloud conditions by year.

(August or September) than later (December, January, or February).

- A relationship between time lost to clouds and the El Niño weather pattern is apparent, but there is significant scatter about the best fit relationship.

\section{References}

[KPNO-CTIO (1966)] 1966, AJ, 71, 229

[Bernstein et al. (2012)] Bernstein, J. P., Kessler, R., Kuhlmann, S., et al. 2012, ApJ, 753, 152

[CTIO (2015)] Cerro Tololo Inter-American Observatory 2015, "Was your night photometric?" (La Serena, Chile: NOAO) https://www . ctio. noao.edu/site/phot/sky_ conditions.html

[Diehl et al. (2014)] Diehl, H. T., Abbott, T. M. C., Annis, J., et al. 2014, Proc. SPIE, 9149, 91490V

[Flaugher (2005)] Flaugher, B. 2005, International Journal of Modern Physics A, 20, 3121

[Flaugher et al. (2015)] Flaugher, B., Diehl, H. T., Honscheid, K., et al. 2015, arXiv:1504.02900

[Frieman (2014)] Frieman, J. 2014, Physics Today, 67, 4 


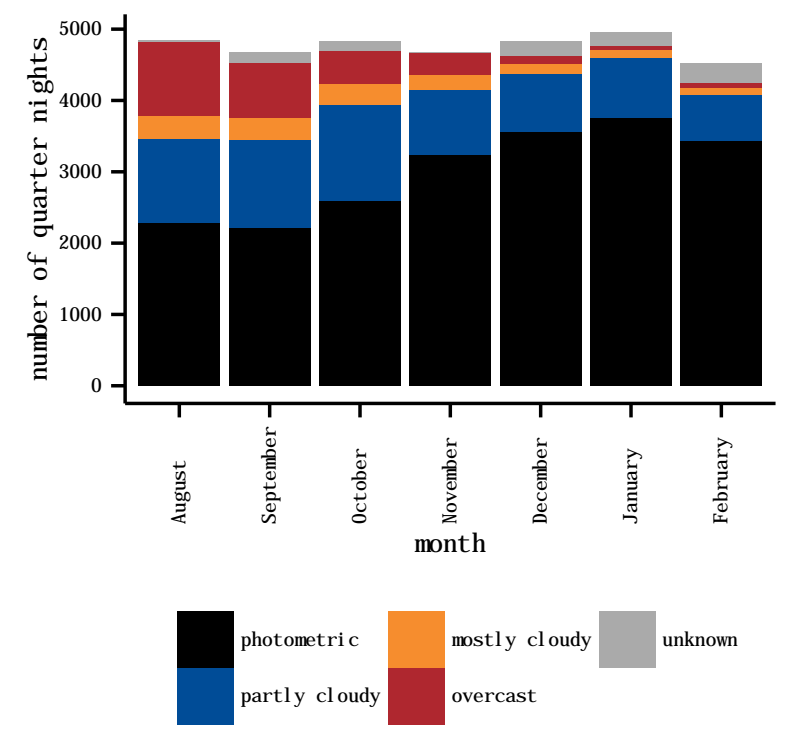

Figure 4: The distribution of cloud conditions by month, combining data from all available years.

[McGill, Tukey, \& Larsen (1978)] McGill, R., Tukey, J. W. and Larsen, W. A., 1978, The American Statistician 32, 12-16

[Neilsen et al. (2015)] Neilsen et al., 2015, (in prep.)

[NOAA CPC (2014)] Climate Prediction Center 2014, El Niño/Southern Oscillation (ENSO) Diagnostic Discussion (College Park, MD: NOAA) http://www.cpc.ncep.noaa.gov/products/analysis_ monitoring/enso_advisory/index.shtml

[NOAA CPC(2014)] Climate Prediction Center 2014, Historical Information (College Park, MD: NOAA) http://www.cpc.ncep.noaa.gov/products/analysis_ monitoring/ensostuff/detrend.nino34.ascii.txt

\section{Acknowledgments}

I am grateful to the CTIO Telescope Operations Group for recording, compiling, and distributing the cloud-cover data on which this memo is based.

William Wester provided valuable discussion and feedback which improved this study.

Funding for the DES Projects has been provided by the U.S. Department of Energy, the U.S. National Science Foundation, the Ministry of Science and Education of Spain, the Science and Technology Facilities Council of the United
Kingdom, the Higher Education Funding Council for England, the National Center for Supercomputing Applications at the University of Illinois at Urbana-Champaign, the Kavli Institute of Cosmological Physics at the University of Chicago, Financiadora de Estudos e Projetos, Fundacao Carlos Chagas Filho de Amparo a Pesquisa do Estado do Rio de Janeiro, Conselho Nacional de Desenvolvimento Cientifico e Tecnologico and the Ministerio da Ciencia e Tecnologia, the Deutsche Forschungsgemeinschaft and the Collaborating Institutions in the Dark Energy Survey.

The Collaborating Institutions are Argonne National Laboratories, the University of California at Santa Cruz, the University of Cambridge, Centro de Investigaciones Energeticas, Medioambientales y Tecnologicas-Madrid, the University of Chicago, University College London, the DES-Brazil Consortium, the Eidgenoessische Technische Hochschule (ETH) Zurich, Fermi National Accelerator Laboratory, the University of Edinburgh, the University of Illinois at UrbanaChampaign, the Institut de Ciencies de l'Espai (IEEC/CSIC), the Institut de Fisica d'Altes Energies, the Lawrence Berkeley National Laboratory, the Ludwig-Maximilians Universitat and the associated Excellence Cluster Universe, the University of Michigan, the National Optical Astronomy Observatory, the University of Nottingham, the Ohio State University, the University of Pennsylvania, the University of Portsmouth, SLAC National Laboratory, Stanford University, the University of Sussex, and Texas A\&M University.

Fermilab is operated by Fermi Research Alliance, LLC under Contract No. De-AC02-07CH11359 with the United States Department of Energy. 


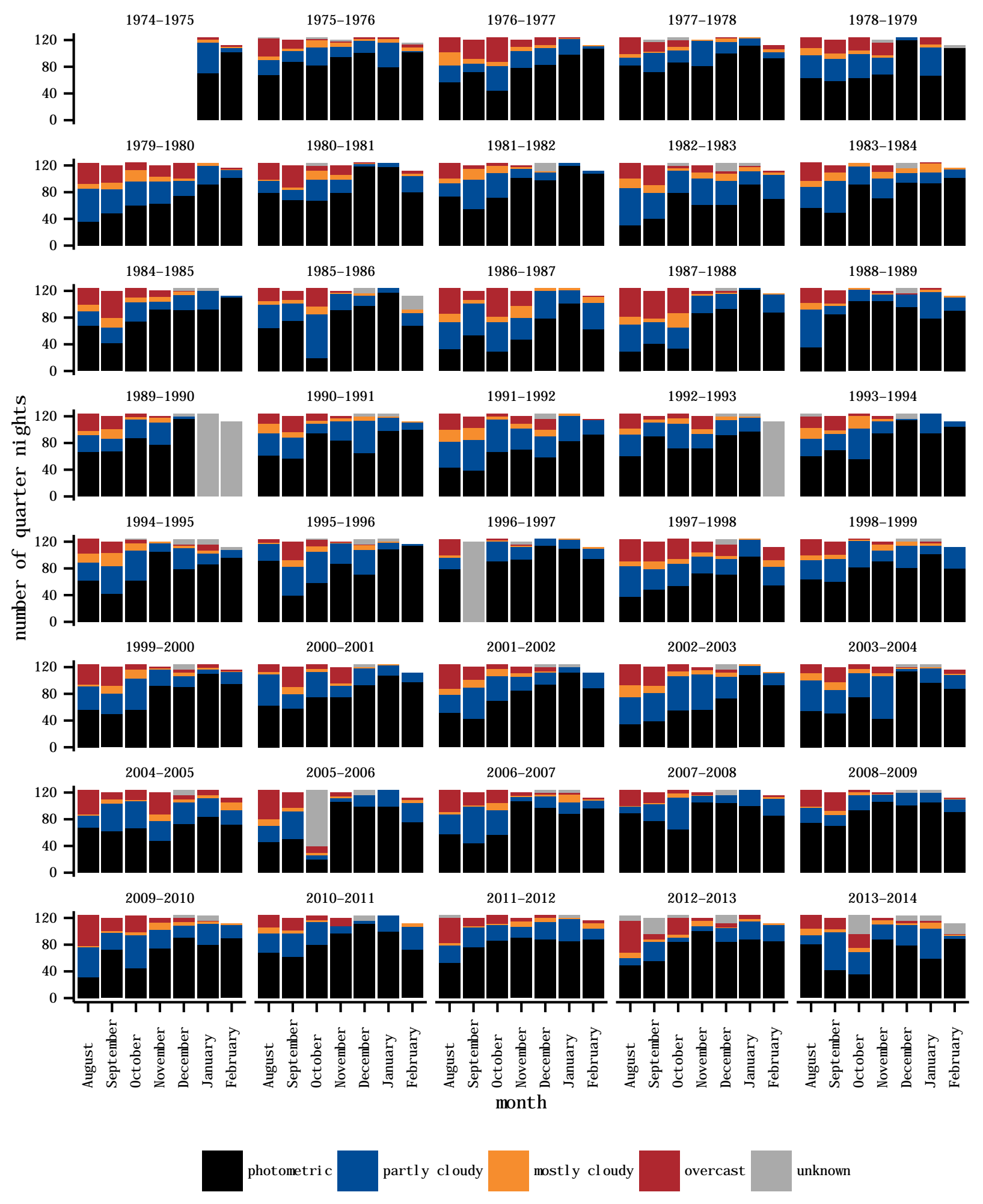

Figure 5: The distribution of cloud conditions by month and DES observing season. 


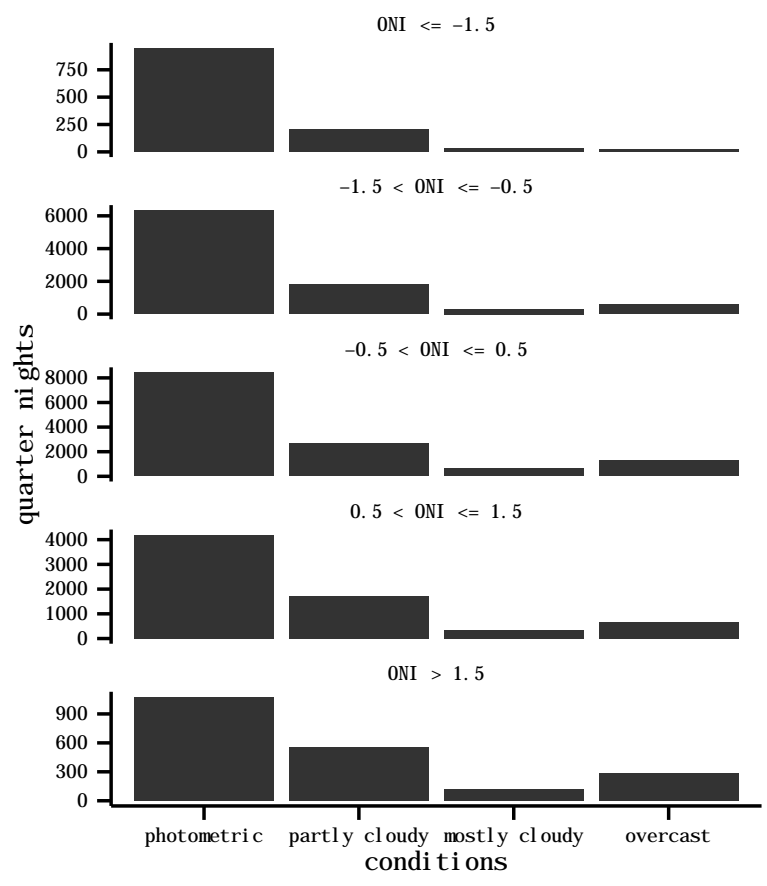

Figure 6: The distribution of cloud conditions by Oceanic Niño Index (ONI).

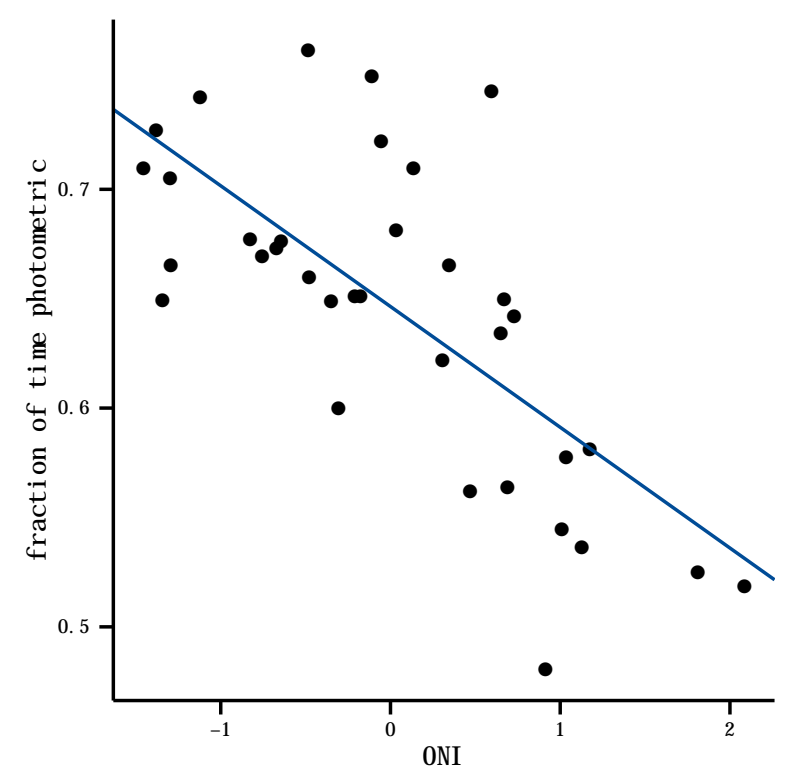

Figure 7: The number of photometric quarter nights from August though February plotted against the mean Oceanic Niño Index (ONI), excluding years with large numbers of quarter nights with no recorded data. 